\title{
Seminal plasma PSA in spinal cord injured men: a preliminary report
}

\author{
Klaus Brasso ${ }^{1}$, Jens Sønksen ${ }^{1}$, Peter Sommer ${ }^{1}$, Lars Ødum ${ }^{3}$, Fin Biering-Sørensen ${ }^{2}$, Peter Iversen ${ }^{1}$ and \\ Jørgen Kvist Kristensen ${ }^{1}$ \\ ${ }^{1}$ Department of Urology and ${ }^{2}$ Centre for Spinal Cord Injured, Rigshospitalet, University of Copenhagen; ${ }^{3}$ Department \\ of Clinical Biochemistry, Bispebjerg Hospital, University of Copenhagen, Denmark
}

\begin{abstract}
Objective: The aim of the study was to evaluate the impact of spinal cord injury on seminal plasma PSA concentration.

Material: Semen obtained from normal controls $(n=20)$ and patients $(n=16)$ with spinal cord injury.

Results: The concentration of prostate specific antigen in seminal plasma depended on the level of spinal cord injury, with significantly lower concentrations in patients with spinal cord lesions below T7 compared to both normal age-matched controls $(P=0.007)$ and patients with spinal cord injuries at or above T7 $(P=0.017)$.

Conclusions: The results add to the previously reported observations of the possible impact of neurogenic stimulation on prostate activity. More studies of neurogenic stimulation in prostate growth and development are warranted to further elucidate the possible impact of neurogenic factors in the development of prostatic diseases.
\end{abstract}

Keywords: Prostate specific antigen; semen; seminal plasma; spinal cord injuries

\section{Introduction}

Prostate specific antigen (PSA) was first isolated from human seminal plasma. ${ }^{.}$PSA is produced almost exclusively in the prostate epithelium and is normally regarded as an indicator of the activity in the prostate gland. Furthermore, changes in PSA measured in serial serum samples correlate to the development of prostatic disease. ${ }^{2}$ Prostate development and growth depends on normal androgen supply. ${ }^{3}$ In vitro studies in animal models suggest that sympathetic activity may induce growth and/or maintain cell survival in the prostate ${ }^{4}$ and human prostate cancer cell proliferation is inhibited by alphaadrenergic blocking agents. ${ }^{5}$

The aim of this study was to evaluate the seminal plasma PSA concentrations in patients with spinal cord injuries (SCI) compared to normal controls, and to evaluate the impact of level of spinal cord injury on seminal plasma PSA-concentration.

\section{Material and methods}

Semen was obtained from 20 normal, healthy and fertile controls following masturbation and $16 \mathrm{SCI}$ patients undergoing either vibratory or electrostimulation to induce ejaculation as described by Sønksen et $a l^{6}$ and $\mathrm{Ohl}$ et $a l^{7}$ Details about the patients included are given in Table 1. Before assisted ejaculation procedures a urine culture was performed in all SCI patients. Semen was frozen at $-20^{\circ} \mathrm{C}$ within $30 \mathrm{~min}$

Correspondence: K Brasso, Department of Urology D-2111 Rigshospitalet, University of Copenhagen, Blegdamsvej 9, DK-2100 Copenhagen, Denmark after ejaculation. For PSA measurement, semen was diluted $(1: 10000$ or $1: 100000)$ in PSA-negative female serum and analyzed with the automated PSA method from Abbott Labs., Abbott Park, IL ${ }^{8,9}$ performed on an AxSYM (Abbott). ${ }^{10}$ Follicle stimulating hormone (FSH), luteinising hormone (LH) and testosterone levels were measured in SCI patients, but not in controls. Seminal plasma PSA concentrations were compared using the Mann-Whitney rank sum test. $P$ values less than 0.05 are considered significant.

\section{Results}

The age and seminal plasma PSA concentrations in controls and SCI patients are shown in Table 2. No significant differences in age, completeness of SCI, or time since injury were found between patients with spinal cord lesions at or above T7 compared to lesions below T7. All SCI patients had normal FSH, LH and testosterone levels. No SCI patients had significant bacterial growth in the urine before the ejaculation procedures.

The seminal plasma PSA concentrations were lower in patients with SCI compared to normal age-matched controls, however the difference did not reach a significant level, $(P=0.08)$. No difference in seminal plasma PSA was found between patients with lesions at or above $\mathrm{T} 7$ and normal controls.

Seminal plasma PSA concentrations in patients with lesions below T7 were significantly lower than concentrations found in controls, $(P=0.007)$, and in patients with lesions at or above $\mathrm{T} 7,(P=0.017)$. No 
Table 1 Spinal cord injured patients

\begin{tabular}{|c|c|c|c|c|c|}
\hline Age & $\begin{array}{l}\text { Duration } \\
\text { of injury } \\
\text { (years) }\end{array}$ & \multicolumn{2}{|c|}{ Level of lesion } & Method & $\begin{array}{c}\text { Seminal } \\
P S A \text { conc. } \\
(m g / L)\end{array}$ \\
\hline \multicolumn{6}{|c|}{ Lesions at or above $\mathrm{T} 7$} \\
\hline 31 & 9 & $\mathrm{C} 2$ & Compl. & PVS & 892 \\
\hline 28 & 10 & $\mathrm{C} 5$ & Compl. & PVS & 271 \\
\hline 29 & 6 & $\mathrm{C} 5$ & Compl. & PVS & 440 \\
\hline 33 & 13 & C6 & Compl. & PVS & 1534 \\
\hline 21 & 4 & C6 & Compl. & PVS & 1437 \\
\hline 31 & 12 & $\mathrm{C} 7$ & Compl. & PVS & 243 \\
\hline 31 & 29 & T6 & Compl. & EEJ & 780 \\
\hline 32 & 2 & T6 & Compl. & PVS & 330 \\
\hline 36 & 1 & $\mathrm{~T} 7$ & Incompl. & PVS & 440 \\
\hline 45 & 29 & $\mathrm{~T} 7$ & Compl. & PVS & 480 \\
\hline \multicolumn{6}{|c|}{ Lesions below T7 } \\
\hline 44 & 18 & T8 & Compl. & EEJ. & 140 \\
\hline 27 & 7 & $\mathrm{~T} 8$ & Compl. & EEJ. & 110 \\
\hline 42 & 20 & T12 & Compl. & EEJ. & 944 \\
\hline 34 & 12 & $\mathrm{~T} 12$ & Compl. & EEJ. & 46 \\
\hline 25 & 9 & T12 & Incompl. & EEJ. & 108 \\
\hline 29 & 7 & $\mathrm{~T} 12$ & Compl. & EEJ. & 71 \\
\hline
\end{tabular}

PVS: Penile vibratory stimulation. EEJ: Electroejaculation

Table 2 Age and seminal plasma PSA concentrations in controls and SCI patients

\begin{tabular}{lrcc}
\hline & Age (years) & $\begin{array}{c}\text { Seminal PSA } \mathrm{mg} / \mathrm{L} \\
\text { Median/range }\end{array}$ & \begin{tabular}{c} 
Median $/$ range \\
\hline Controls
\end{tabular} \\
$\begin{array}{l}\text { Patients } \\
\text { At or above T7 }\end{array}$ & 10 & 34 years $/ 25-48$ & $535 / 238-2379$ \\
Below T7 & 6 & 32 years $/ 21-45$ & $460 / 243-1534$ \\
\hline
\end{tabular}

significant differences in seminal plasma PSA concentrations were found, when comparing ejaculates obtained by either vibratory or electrostimulation.

\section{Discussion}

PSA is secreted in high concentrations by the epithelium in the prostate, and is involved in the liquefaction of the seminal coagulum formed at ejaculation. ${ }^{11}$ Seminal plasma PSA concentration is approximately $10^{5}-10^{6}$ times higher than PSA concentration in serum and the majority of PSA in seminal plasma is present in the catalytically active single-chain form. ${ }^{12}$

Prostate growth and development of prostatic diseases depends on age and androgen supply. ${ }^{3}$ Other factors, however, may modify the impact of androgens, such as a low 5-alpha-reductase activity found in Japanese men, which may be part of the explanation for the lower prostate cancer incidence in Japan. ${ }^{13}$
The prostate normally contains a number of neuroendocrine cells and it has been suggested, that neuroendocrine stimulation may affect prostate growth and differentation. ${ }^{14,15}$ The number of neuroendocrine cells in the prostate is increased in benign prostatic hyperplasia compared to normal glands. ${ }^{14}$ Further, neuroendocrine cells can be found in the majority of prostatic carcinomas. ${ }^{15}$ The neuroendocrine cells are often found in close relation to afferent nerves and produce a number of bioactive hormone-related substances of which some act as growth stimulators, ${ }^{16}$ while others like somatostatin can inhibit trophic or growth factor activity. ${ }^{15}$

The neurogenic stimulation of the male accessory sexual glands including the prostate arises from sympathetic nerves from the thoracolumbar spinal cord (T11-L2) and from parasympathetic nerves from the sacral spinal cord $(\mathrm{S} 2-4) .{ }^{17,18}$ When analyzing the impact of the SCI level we found seminal plasma PSA concentrations to be significantly lower in patients with lesions below T7 compared to patients with lesions at or above this level. More logically a spinal cord cut-off level at T10 would have been expected. However, previously it has been demonstrated, that seminal somatostatin levels in patients with SCI depended on the level of spinal cord injury, with significantly higher levels in patients with lesions below T6. ${ }^{19}$ This finding suggests that the neurogenic stimulation of somatostatin is dependent on nerve fibres arising above this level. In addition, semen analyses from SCI men have demonstrated significantly better sperm motility in patients with lesions at or above T6 compared to lesions below this level. ${ }^{20}$ PSA is in our study lower, although not significantly, in patients with spinal cord injuries compared to healthy controls. It is noteworthy that patients with normal testosterone levels and SCI below T7 had significantly lower seminal PSA than both normal controls and SCI patients with lesions at or above T7. This finding suggests that some neurogenically mediated factor plays a role in the PSA production. The inverse correlation between high somatostatin and low seminal plasma PSA concentrations found in patients with spinal cord lesions below the level of T6/ T7 suggests that this spinal cord level represents a crucial point in the neurogenic stimulation of the prostate gland.

PSA has been demonstrated to degrade, when serum samples are stored. ${ }^{21}$ Most likely a similar degradation will occur in seminal plasma when these samples are stored. The observed difference found in seminal plasma PSA concentration, however, is unlikely to be explained by storage, as the handling of samples were identical in the two groups. Serum PSA concentration increases in patients with urinary retention, lower urinary tract infections, and prostatitis, ${ }^{22,23}$ conditions frequently present in patients with SCI. The impact of these conditions on seminal plasma PSA has not been elucidated. However, if seminal plasma PSA were to increase, this would tend to diminish the difference in 
seminal plasma PSA concentrations by producing higher seminal plasma PSA in the patients with SCI.

Serum PSA concentrations have been demonstrated to vary when measurements are repeated. ${ }^{24}$ To what extent seminal plasma PSA concentration varies is not known, and studies including more patients and repeated measurements are ongoing.

Further studies on the possible effect of neurogenic stimulation of prostatic development and growth are warranted, and may possibly add to our understanding of both neuroendocrine differentiation and aetiological factors in the development of prostatic diseases.

\section{References}

1 Sensabaugh GF. Isolation and characterization of a seminalspecific protein from human seminal plasma: a potential new marker for semen identification. J Forensic Sci 1978; 23: $106-$ 115.

2 Carter $\mathrm{HB}$ et al. Longitudinal evaluation of prostate-specific antigen levels in men with and without prostate disease. JAMA 1992; 267: $2215-2220$.

3 Bostwick DG et al. The association of benign prostatic hyperplasia and cancer of the prostate. Cancer 1992; 70: $291-$ 301.

4 Yang $\mathrm{G}$ et al. Induction of apoptosis by doxazosin in the BPHmouse prostate reconstruction model. Eur Urol 1996; 30 (suppl.2) Abstract 41.

5 Allen LF, Fergusson SB, Moore ME. Prostate cancer cell growth inhibition by doxazosin. Eur Urol 1996; 30 (suppl.2) Abstract 73.

6 Sønksen J, Biering-Sørensen F, Kristensen JK. Ejaculation induced by penile vibratory stimulation in men with spinal cord injuries. The importance of the vibratory amplitude. Paraplegia 1994; 32: $651-660$.

7 Ohl DA et al. Predictors of success in electroejaculation of spinal injured men. J Urol 1989; 142: 1483-1486.

$8 \mathrm{Yu} \mathrm{H}$, Diamandis EP. Prostate-specific antigen in milk of lactating women. Clin Chem 1995; 41: 54-58.

9 Oesterling JE, Moyad MA, Wright GL Jr, Beck GR. An analytical comparison of the three most commonly used prostate-specific antigen assays: Tandem-R, Tandem-E and IMX. Urology 1995; 46: 524-532.
10 Costrongs GM et al. Evaluation of the Abbott automated random, immediate and continuous access immunoassay analyser, the AxSYM. Eur J Clin Chem Clin Biochem 1995; 33: $105-111$.

11 Lilja H. A kallikrein-like serine protease in prostatic fluid cleaves the predominant seminal vesicle protein. J Clin Invest 1985; 76: $1899-1903$.

12 Abrahamsson P-A, Lilja H, Oesterling JE. Molecular forms of serum PSA; the clinical value of percent free prostate-specific antigen. Urol Clin N Am 1997; 24: 353-365.

13 Ross R, Bernstein L, Lobo RA et al. 5-alpha-reductase activity and risk of prostate cancer among Japanese and US white and black males. Lancet 1992; 339: 887-889.

14 Abrahamsson P-A et al. Peptide-hormone- and serotoninimmunoreactive cells in normal and hyperplastic prostate glands. Path Res Pract 1986; 181: 675-683.

15 Di Sant'Agnese PA. Neuroendocrine differentiation in human prostatic carcinoma. Hum Pathol 1992; 230: 287-296.

16 Abrahamsson P-A, Gershagen S. Neuroendocrine differentiation and hormone-refractory prostate cancer. In: Abrahamsson P-A and Iversen P. (eds). Proceedings from International conference on prostate carcinoma, Copenhagen 1995. 1995 Wells Medical Limited, Kent, UK.

17 Sønksen J, Biering-Sørensen F. Fertility in men with spinal cord or cauda equina lesions. Semin Neurol 1992; 12: 106-114.

18 Lecthenberg R, Ohl DA. Normal sexual function. In: Lechtenberg R, Ohl DA (eds). Sexual dysfunction. Lea and Febiger. Philidelphia 1994.

19 Ødum L, Sønksen J, Biering-Sørensen F. Seminal somatostatin in men with spinal cord injury. Paraplegia 1995; 33: $374-376$.

20 Sønksen J et al. Quality of semen obtained by penile vibratory stimulation in men with spinal cord injuries: observations and predictors. Urology 1996; 48: 453 - 457.

21 Stenman UH et al. Serum concentrations of prostate specific antigen and its complex with alpha-1-antichymotrypsin before diagnosis of prostate cancer. Lancet 1994; 344: 1954-1958.

22 Tchetgen MBN, Oesterling JE. The effect of prostatitis, urinary retention, ejaculation, and ambulation on the serum prostatespecific antigen concentration. Urol Clin N Am 1997; 24: $283-$ 291.

23 Neal DJ Jr, Clejan S, Sarma D, Moon TD. Prostate specific antigen in prostatitis I. Effect on serum PSA in human and nonhuman primate. Prostate 1992; 20: $105-111$

24 Prestigiacomo AF, Stamey TA. Physiological variation of serum prostate specific antigen in the 4.0 to $10.0 \mathrm{ng} / \mathrm{ml}$. range in male volunteers. J Urol 1996; 155: $1977-1980$. 